\title{
Extreme ultraviolet interference lithography at the Paul Scherrer Institut
}

\author{
Vaida Auzelyte \\ Christian Dais \\ Patrick Farquet \\ Paul Scherrer Institut \\ Laboratory for Micro- and Nanotechnology \\ Villigen 5232, Switzerland \\ E-mail: vaida.auzelyte@psi.ch
}

Detlev Grützmacher

Institute of Bio- and Nanosystems 1

Research Center of Jülich

52425 Jülich, Germany

\author{
Laura J. Heyderman \\ Feng Luo \\ Paul Scherrer Institut \\ Laboratory for Micro- and Nanotechnology \\ Villigen 5232, Switzerland
}

\section{Sven Olliges}

Swiss Federal Institute of Technology (ETH) Zurich

Laboratory for Nanometallurgy

Department of Materials

Wolfgang-Pauli-Strausse 10

Zurich, $\mathrm{CH}-8093$, Switzerland

\section{Celestino Padeste \\ Pratap K. Sahoo}

Paul Scherrer Institut

Laboratory for Micro- and Nanotechnology

Villigen 5232, Switzerland

\section{Tom Thomson}

University of Manchester Oxford Road

School of Computer Science

Kilburn Building

Manchester M13 9PL, United Kingdom

\section{Andrey Turchanin}

University of Bielefeld

Faculty of Physics

Physics of Supermolecular Systems

D-33615 Bielefeld, Germany

\section{Christian David}

Paul Scherrer Institut

Laboratory for Micro- and Nanotechnology

Villigen 5232, Switzerland

\author{
Harun H. Solak \\ Paul Scherrer Institut \\ Laboratory for Micro- and Nanotechnology and \\ Eulitha AG \\ Villigen 5232, Switzerland
}

\begin{abstract}
We review the performance and applications of an extreme ultraviolet interference lithography (EUV-IL) system built at the Swiss Light Source of the Paul Scherrer Institut (Villigen, Switzerland). The interferometer uses fully coherent radiation from an undulator source. 1-D (line/ space) and 2-D (dot/hole arrays) patterns are obtained with a transmission-diffraction-grating type of interferometer. Features with sizes in the range from one micrometer down to the 10-nm scale can be printed in a variety of resists. The highest resolution of 11-nm half-pitch line/space patterns obtained with this method represents a current record for photon based lithography. Thanks to the excellent performance of the system in terms of pattern resolution, uniformity, size of the patterned area, and the throughput, the system has been used in numerous applications. Here we demonstrate the versatility and effectiveness of this emerging nanolithography method through a review of some of the applications, namely, fabrication of metallic and magnetic nanodevice components, self-assembly of $\mathrm{Si} / \mathrm{Ge}$ quantum dots, chemical patterning of self-assembled monolayers (SAM), and radiation grafting of polymers. () 2009 Society of Photo-Optical Instrumentation Engineers. [DOI: 10.1117/1.3116559]
\end{abstract}

Subject terms: nanolithography; extreme ultraviolet; interference lithography; metal nanowire; quantum dot; magnetic nanostructure; self-assembled monolayer; polymer grafting.

Paper 08163SSR received Oct. 26, 2008; revised manuscript received Feb. 5, 2009; accepted for publication Mar. 2, 2009; published online Apr. 27, 2009.

\section{Introduction}

Extreme ultraviolet interference lithography (EUV-IL) is a newly emerging nanolithography method that combines the advantages of a parallel fabrication process with high resolution. These features make it an attractive tool for researchers who are increasingly in need of a nanopatterning capability that is beyond what is available from other methods such as photolithography, electron beam lithography (EBL), and scanning probe lithography, in terms of resolution or throughput. After early interference lithography experiments performed in the vacuum UV1 and EUV regions ${ }^{2}$ dedicated EUV-IL tools have been set up using synchrotron 14 and laser sources 5 Such tools using plasmabased EUV sources are also being considered

In most reported EUV-IL work, the wavelength of choice has been $13.4 \mathrm{~nm}$. The reason behind this choice is manifold. First, the possible next-generation EUV lithography (EUVL) technology that is being developed for fabrication of future semiconductor devices uses this wavelength. The EUV-IL method has played an important role in the development of high-resolution resists for this technol-

$1932-5150 / 2009 / \$ 25.00$ ๑ 2009 SPIE 
ogy by making high-resolution exposures ayailable long before EUV projection tools become available 99 Second, it is relatively easy to fabricate diffraction gratings with sufficiently high diffraction and transmission efficiency at this wavelength on silicon nitride membranes. Finally, the short inelastic mean free path of photoelectrons created by EUV photons in a resist at this energy $(92.5 \mathrm{eV})$ means that the blurring effect of photoelectrons will be insignificant down to sub-10 nm resolution. Of course, we should also mention that this wavelength allows an ultimate half-pitch (hp) resolution of below $4 \mathrm{~nm}$, which can be as small as a quarter of the wavelength $(\lambda)$ :

$\mathrm{hp}=\frac{\lambda}{4 \sin (\theta / 2)}$,

where $\theta$ is the angle between two diffracted beams.

Several different EUV-IL schemes have been realized or proposed. A Lloyd's mirror interferometer, which is a wavefront division method, was used to expose linear gratings and 2-D patterns in a double exposure scheme 510 11 Amplitude division interferometers, one involying beamsplitter gratings with two recombining mirrors 12 and another that has two cascaded gratings, 13 were also recently demonstrated. The EUV-IL system at the Paul Scherrer Institut (PSI) in Villigen, Switzerland 4 whose application is the subject of this review, 1 ses a diffraction-grating-based wavefront division scheme 23 We describe this scheme in more detail in the following section. In addition to two and multiple beam interference methods, a holographic approach was also demonstrated?

\section{Extreme Ultraviolet Interference Lithography System at the Paul Scherrer Institut}

The EUV-IL system at the XIL beamline of the Swiss Light Source (SLS) uses light that is generated by a 42-pole undulator with a $212 \mathrm{~mm}$ period, which is inserted into the $2.4 \mathrm{GeV}$ electron storage ring. The SLS, as a third generation synchrotron, is constructed to provide a spatially coherent beam for photon energies of up to about $100 \mathrm{eV}$, thanks to the small size and divergence of the electron beam 14 The spatial coherence is essential for the basic wavefront division interference scheme that is used at this beamline. The temporal/longitudinal coherence of the light emanating from the source is related to the spectral bandwidth $\Delta \lambda / \lambda$. For an undulator, this is related to the number of magnetic poles, which translates to a rather large spectral width of about $2.5 \%$ for our source. To get a narrower bandwidth, the beam can be filtered using a monochromator. However, this leads to a significant loss of photon flux. The diffraction-grating-based interference schemes described next are achromatic. Therefore in most experiments the interferometric exposures are performed using the full bandwidth of the undulator radiation without additional spectral filtering. However, the beam is filtered by a pinhole to guarantee full spatial coherence at the interferometer. The resulting beam at the interferometer has a width of about 3 to $5 \mathrm{~mm}$ [full width at half maximum (FWHM)], which depends on the diameter of the pinhole used for spatial filtering. The photon flux incident on the interferometer is stable, thanks to the stability of the source

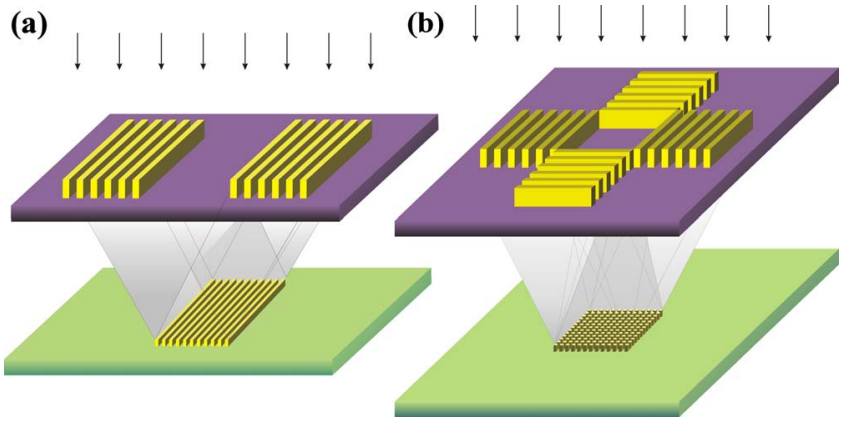

Fig. 1 The interference schemes of two- and four-grating designs to expose line and hole/dot patterns, respectively.

and the beamline optics, as well as the top-up mode of operation of the SLS storage ring. With the typical flux of 20 to $50 \mathrm{~mW} / \mathrm{cm}^{2}$, dot and line patterns in polymethyl methacrylate (PMMA) resist can be exposed within $10 \mathrm{sec}$. The beam is switched on and off by a fast mechanical shutter placed in front of the exposure chamber. Substrates ranging from several square centimeters chips up to $200 \mathrm{~mm}$ wafers can be exposed.

The basic interference scheme that is used at the PSI system is illustrated in Fig. 1. The spatially coherent incident beam is diffracted by linear gratings that are patterned on a single, partially transparent membrane. The resulting mutually coherent beams interfere at a certain distance along the beam direction. The interference fringes are used to expose a pattern in a resist film. The schemes in Fig. 1 create periodic patterns that have half the period of the interferometer grating. The size and shape of each exposure pattern is the same as the size and shape of the grating. Pairs of diffraction gratings with different periods can be placed on a single mask and the resulting pattern printed simultaneously in one exposure step. In this way, patterns from the micrometer down to the nanometer scale can be created in a single exposure in EUV-IL.

Two linear gratings are used to create lines, while hole/ dot patterns can be made in one exposure step by the interference of three or more diffracted beams (Fig. 1, 15 More complex structures such as rings and crosses can be made by eight-beam interference 16 Depending on the design of the mask, the relation between the period of the diffraction gratings and the fringe pattern varies. For example, in a four-beam scheme, demagnification factors of 2 or $\sqrt{2}$ (in terms of pattern period) are obtained depending on the relative phases of the interfering beams 16 This demagnification factor is very helpful for the mask fabrication. For example, $200 \mathrm{~nm}$ period gratings written on a mask will expose $100 \mathrm{~nm}$ period lines on the sample. Examples of two- and four-beam masks mounted on stainless steel rings are shown in Fig. 2 The masks are prepared using electron beam lithography (EBL) and reactive ion etching pattern transfer processes on $100-\mathrm{nm} \mathrm{Si}_{3} \mathrm{~N}_{4}$ membranes coated with a thin $\mathrm{Cr}$ film. 17 The gratings are written in a resist with EBL and later etched into the $\mathrm{Cr}$ film. The thickness of the grating metal layer, the grating duty cycle, and the material (e.g., $\mathrm{Cr}$ ) determine the EUV diffraction efficiency. Masks fabricated in the same way have been also used in other EUV-IL systems 313 


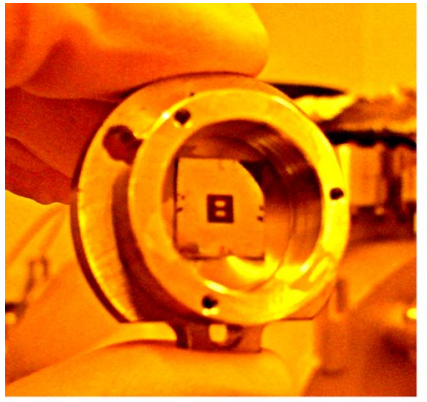

(a)

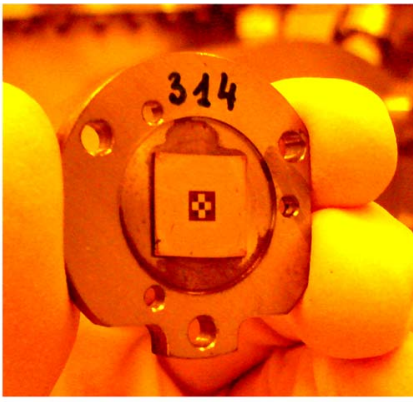

(b)
Fig. 2 (a) Two-beam and (b) four-beam interference masks prepared for exposures. The masks consist of a square silicon chip on frame with a $3 \times 3 \mathrm{~mm}^{2} \mathrm{Si}_{3} \mathrm{~N}_{4}$ membrane in the center where the gratings are patterned.

Different periods and patterns can be included on a single mask. As an example, the layout of a mask that is designed to expose sets of $800 \mu \mathrm{m}$-long lines with six different half-pitches in the range of 20 to $50 \mathrm{~nm}$ is shown in Fig. 3(a) In addition, these long lines can be cleaved in parallel to obtain cross sectional images, and the availability of many different line widths in a single exposure greatly facilitates this resist characterization process. This mask was designed for testing experimental resists for EUVL, the leading candidate mentioned in the 2007 International Technology Roadmap for Semiconductors (ITRS) as one of the nexy-generation lithography technologies for the $32 \mathrm{~nm}$ node 18 One of the challenges is the development of dedicated EUV resists, which are expected to allow the printing of $22 \mathrm{~nm}$ and smaller node structures, and simultaneously meet the requirements for line-edge roughness and dose. The SEM image of a pattern exposed using this mask in a EUV resist can be seen in Fig. 3(b) The EUV-IL system at PSI has been used extensively for screening these resists 91920

High-resolution electron beam resists, such as PMMA, calixerene, and hydrogen silsesquioxane (HSQ) have been (a)

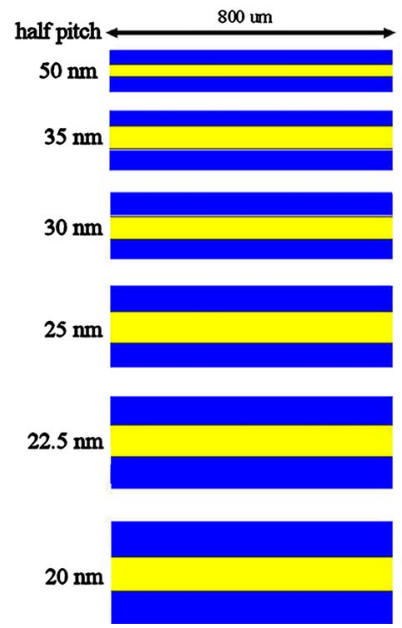

(b)

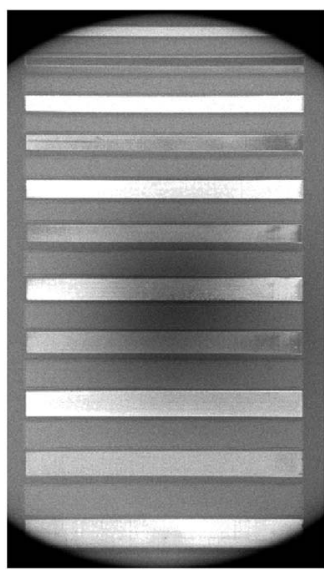

Fig. 3 The layout of a 20 to $50 \mathrm{~nm}$ half-pitch line mask and scanning electron microscopy (SEM) image of its pattern exposed into EUV resist. The interference areas are large and homogenous, suitable for producing cross sections for SEM characterization.

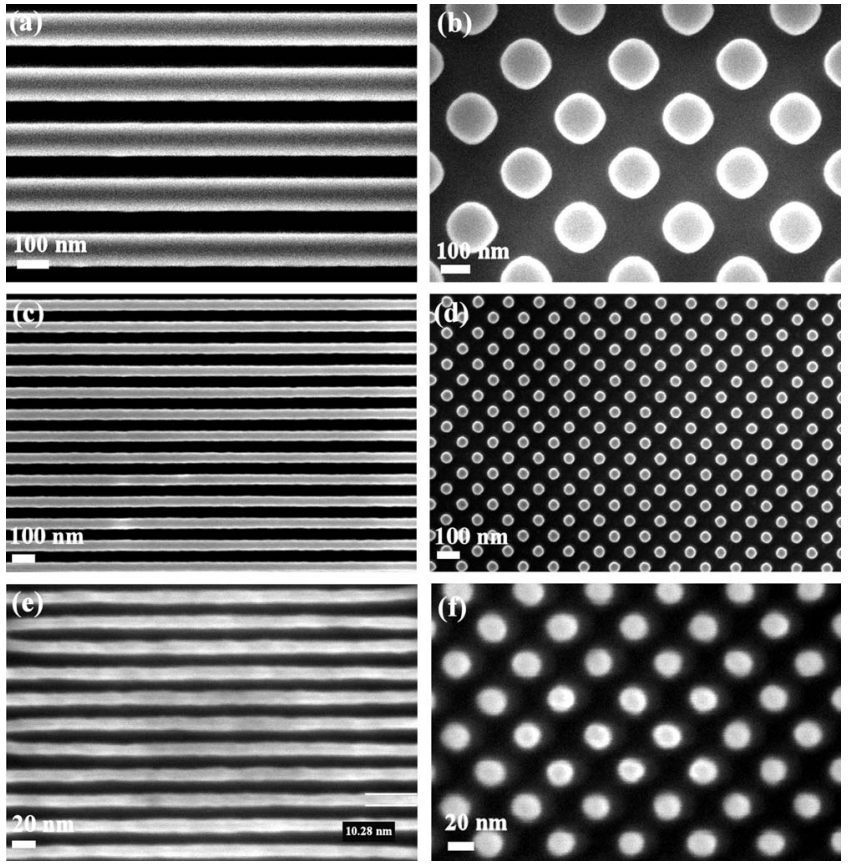

Fig. 4 Line and dot patterns made by EUV-IL exposure in HSQ using different masks: (a) $125 \mathrm{~nm}$ half-pitch lines, (b) $175 \mathrm{~nm}$ halfpitch dots, (c) and (d) $50 \mathrm{~nm}$ half-pitch lines and dots, (e) $11 \mathrm{~nm}$ half-pitch lines, and (f) $19 \mathrm{~nm}$ half-pitch dots.

the resists of choice in EUV-IL applications 10 21/22 There are rapid advances made in the development of chemically amplified EUV resists, and we expect that these resists will also find use in EUV-IL, further enhancing the throughput of this emerging technique.

Examples of line and dot patterns with various periods that were realized using the EUV-IL tool at PSI are demonstrated in Fig. 4. All of the structures in this figure were exposed in negative tone HSQ resist with various thicknesses. Note the excellent pattern definition and the high quality in terms of line edge roughness. The lines in Fig. 4(e) have a half-pitch of $11 \mathrm{~nm}$, which represents the highest resolution pattern reported with a photolithography method and proves the feasibility of obtaining such small patterns with EUV exposures. The resist thickness for this particular pattern was $20 \mathrm{~nm}$. This result lets us conclude that the blurring effects of photo- or secondary-electrons, if any, are not strong enough to prevent us from reaching $11 \mathrm{~nm}$ resolution. Further experiments are underway to explore the ultimate limits of the resolution of EUV exposures.

More advantages of EUV-IL come from the fact that the patterns can be created in a step-and-repeat manner, so that an area of several square millimeters can be exposed in each step. This is considerably faster than conventional focused beam methods, such as EBL or focused ion beam (FIB) lithography. This rapid printing capability allows the production of a large number of samples. Additionally, the mask-based method provides excellent reproducibility, in contrast to serial writing approaches where uniformity problems can be encountered. 

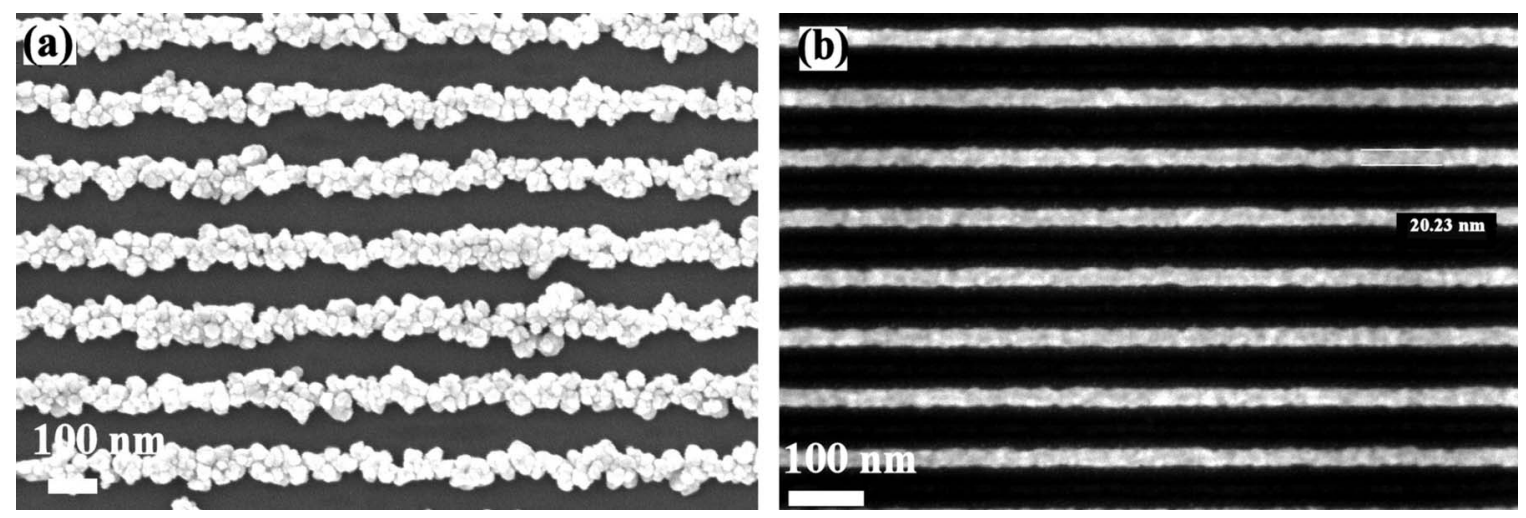

Fig. 5 (a) $100 \mathrm{~nm}$ period nanowires consisting of $20 \mathrm{~nm}$ gold colloids formed on $\mathrm{Nb}_{2} \mathrm{O}_{5}$ substrate, and (b) 25 nm-wide gold nanowires made using a double stack PMMA/HSQ resist process with shadow metal deposition.

\section{Applications}

Having reviewed the basic properties of EUV-IL as a nanolithography method, in this section we present several examples of its use in various research areas. The work was performed using the capabilities of the EUV-IL tool at the PSI over a period of about five years. They demonstrate the versatility of this new method and the use of its distinguishing feature, namely the combination of high resolution and high throughput. We do not report on all of the applications of the EUV-IL method that have been demonstrated using the PSI EUV-IL system, which has also led to the production of nanophotonic devices, 2324 nanoimprint stamps, holographic fabrication of Fresnel zone plates, 26-27 and guided self-assembly of block copolymers and colloidal particles 29 Thanks to its success, EUV-IL technology has entered the commercial phase through a spin-off company of PSI (see http://www.eulitha.com) that makes specialized samples such as commercially available nanoimprint stamps.

\section{Arrays of Metal Nanowires}

At the nanometer scale, physical properties of metallic nanowires are expected to deviate significantly from the bulk metal due to confinement and surface effects. For example, the conductivity of metal nanowires changes considerablydue to the drastic increase of the surface-to-volume ratio 30 Their mechanical properties, such as the yield strength, also change as their diameter is reduced to some tens of nanometers. The novel properties of nanowires make them candidates for use in nanoelectromechanical systems, for example for electronic circuits, data storage, or biomedical applications

EUV-IL has been used to produce large arrays of planar metal nanowires. To study the nanowire properties, one needs to produce a substantial number of nanowires and arrange them on a surface in a controlled way. Besides the outstanding resolution, the EUV-IL method is a suitable patterning technique to produce the required large arrays of periodic and homogenous lines in a wide size range for research on these systems.

The goal of the fabrication effort is to produce nanowire arrays with wire widths in the range from sub-10 $\mathrm{nm}$ down to $100 \mathrm{~nm}$, over areas of the order of several millimetersquare to study their mechanical, electronic, and optical properties. For the fabrication of metallic nanowires, two different approaches were tried: selective assembly of metal colloids on prepatterned substrates and metallization using a modified lift-off process.

In the first method, EUV-IL-created patterns in resists were used as a template to guide the assembly of Au colloids to form nanowires 33 The wires were produced on $\mathrm{Nb}_{2} \mathrm{O}_{5}$-coated surfaces via PLL-g-PEG chemistry. Singlestrand DNA was attached to biotin molecules arranged on $100 \mathrm{~nm}$ period lines. Au colloids of diameters 5, 20, or $100 \mathrm{~nm}$, tagged with the complementary DNA strand, were then selectively attached to the surface. Figure 5(a) shows an SEM image of gold wires composed of $20 \mathrm{~nm}$ colloids, and demonstrates the selectivity of this chemical recognition process, i.e., Au colloids are only found along the molecularly patterned lines. The lines can be used as the basis for sensitive biosensors for label-free electrical and optical detection based on surface plasmons.

The second metallization method uses a modified lift-off process 34 A double resist layer of PMMA/HSQ was exposed with EUV-IL. A line pattern was formed in the top HSQ layer after development. This pattern was then etched into the underlying PMMA layer. In the subsequent step, a metal film was deposited at an oblique angle on top of the resist lines from both sides. The angle of deposition was adjusted so that no metal was deposited in the spaces between the resist lines. Oblique angle of this shadow evaporation also changed the line width and allowed us to control the width of the final metal nanowire. After lift-off in acetone, gold and chromium nanowires with 10 to $70 \mathrm{~nm}$ width and 50 to $100 \mathrm{~nm}$ period were obtained. As an example, $20 \mathrm{~nm}$ gold wires are shown in Fig. 5(b) At the sub-10 nm scale, the nanowire integrity starts to be limited by the material properties, such as roughness of resist lines and the grain size of the deposited metal. The electrical properties of both the solid metal nanowires and colloidal nanowires are currently being explored.

Gold nanowires placed on a flexible substrate and subjected to a tensile load provided a model system to study their mechanical properties. Large area and uniform nanowire arrays are needed for this method to provide statistically significant data. Using both the metallization and colloid assembly methods described before, we produced arrays of 40 to $60 \mathrm{~nm}$-wide and 20 to $30 \mathrm{~nm}$-thick nano- 


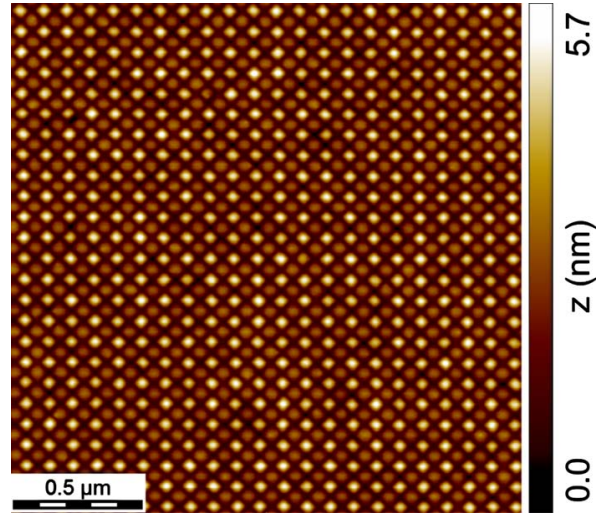

Fig. 6 SFM image of a SiGe QD array with an $x / y$ period of $90 \mathrm{~nm} / 100 \mathrm{~nm}$, respectively.

wires on $125 \mu \mathrm{m}$-thick polyimide substrates. A synchrotron-based $\mathrm{x}$-ray diffraction technique was used to measure their (thermo-) mechanical properties. These experiments showed that gold nanowires are significantly stronger than bulk gold but less strong than the thin film of the same thickness. In contrast to bulk gold, strong temperature-dependent mechanical properties are observed for the nanolines. In-situ SEM investigations revealed an interesting cracking behavior. Line cracks in Au nanolines on homogenous $\mathrm{Cr}$ interlayer thin film were observed at $2.5 \%$ strain, but the same nanolines on hare polyimide substrate crack statistically at $6.5 \%$ strain 3536

\section{SiGe Quantum Dots}

One major drawback of self-assembled quantum dots (QD) lies in their random nucleation, which results in an inhomogeneous distribution of the dot size and position. However, for potentialapplications like the fabrication of QDbased nanodevice 37 or for the demonstration of spintronics and manipulation of qubits, $\stackrel{3839}{39}$ the exact positioning of identical QDs is required. An elegant approach to circumvent the problem of random nucleation is templated selfassembly of semiconductor nanostructures, which combines top-down lithography for definition of the nucleation sites with subsequent epitaxial deposition. Typically, electron beam or optical interference lithography has been used for fabricating prepatterned substrates 4041 However, these methods are limited either in throughput, pattern control, or resolution. In this work EUV-IL was combined with molecular beam epitaxy (MBE) to create $\mathrm{Si} / \mathrm{Ge}$ quantum dot arrays of high resolution with a remarkably narrow size and height distribution.

The process starts with the EUV-IL exposure of PMMAcoated Si(100) substrates. With EUV-IL, hole patterns with periods ranging from 280 down to $35 \mathrm{~nm}$ were generated. Subsequently, the PMMA is developed and the pattern is transferred into the $\mathrm{Si}$ substrate by means of reactive ion etching. The SiGe deposition is performed in a solid source MBE system. The atomic force microscopy (AFM) image in Fig. 6 shows the pattern after MBE deposition of a Si buffer layer and a $\mathrm{Ge}$ dot layer. The $\mathrm{SiGe}$ islands are solely formed in the holes, which results in a highly uniform 2-D array of SiGe QDs. With this technique, perfectly aligned

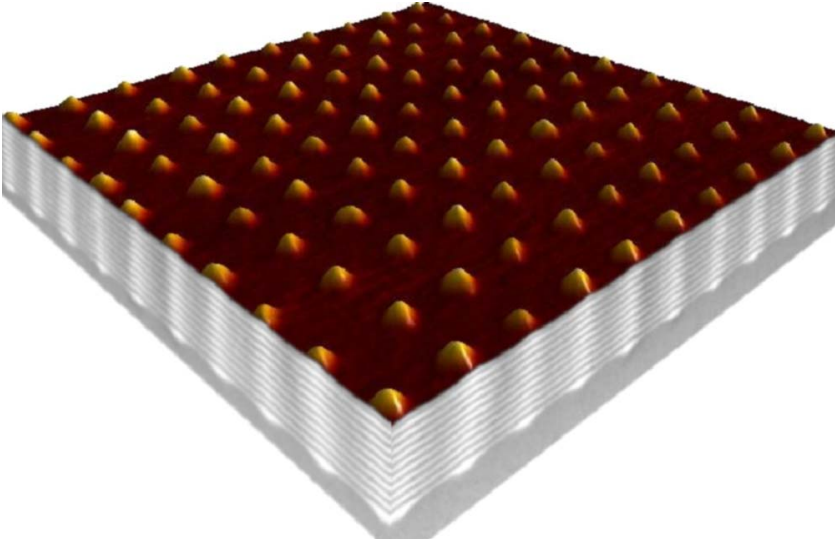

Fig. 7 SiGe QD crystal with a period of $x=90 \mathrm{~nm}, y=100 \mathrm{~nm}$, and $z=11.5 \mathrm{~nm}$. It consists of $11 \mathrm{Ge}$ dot layers separated by $10 \mathrm{Si}$ spacer layers.

SiGe quantum dot arrays with different periods, different QD shapes, and different symmetries have been fabricated 4243 For optical studies, the SiGe quantum dot arrays were investigated by photoluminescence (PL) measurements. Due to the uniformity of the dots, a more efficient PL with much narrower line widths is observed compared to dots grown on unpatterned substrates 44 which is of high interest for the realization of $\mathrm{Si}$ based optoelectronic devices.

Furthermore, the formation of a 3-D QD crystal is realized by subsequently depositing a stack of Ge layers separated by Si spacer layers onto the 2-D SiGe QD array 45 The $\mathrm{Si}$ spacer causes a flattening of the surface for the subsequent SiGe QD growth. During the growth of the Ge layers, the QDs nucleate on top of the buried SiGe QDs-a process induced by the local strain field created by the underlying QDs 46 Figure 7 shows a SiGe QD crystal with a lateral period of $90 \mathrm{~nm}$ in the $x$ direction and $100 \mathrm{~nm}$ in the $y$ direction. The vertical period is $11.4 \mathrm{~nm}$. The image is composed of one AFM and two transmission electron microscopy (TEM) images. Perfect vertical ordering of the SiGe QDs is observed. Moreover, a similarly perfect lateral ordering of the SiGe QDs in the top-most layer is seen in the SFM image of Fig. 7. The islands at the top are (105) faceted and have a square base. X-ray diffraction measurements indicate a high structural quality of the SiGe QD crystals 45 Recently, QD crystals with lateral periods down to $35 \mathrm{~nm}$ have been obtained (unpublished results). It is expected that electronic coupling between adjacent QDs may already occur in such densely packed QD structures. Thus, not only from a structural point of view but also with respect to the band structure, these 3-D ordered QD structures can be regarded as artificial crystals. The EUV-IL technique has been critical to achieve the level of perfection obtained in these samples due to the throughput that allowed the necessary trials on a large number of samples.

\section{Arrays of Magnetic Nanoislands}

The data storage medium in a hard disk drive is currently a granular magnetic thin film where the grain diameters are typically less than $10 \mathrm{~nm}$. Data are written into the medium by a strong, highly confined magnetic field produced by a 


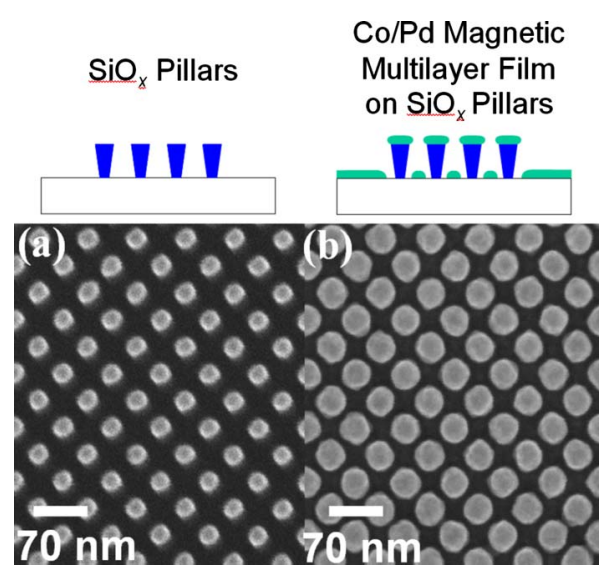

Fig. 8 Schematic diagram of the fabrication of nanoscale magnetic island arrays with EUV-IL and corresponding SEM images of $\mathrm{SiO}_{x}$ pillars with periods of $50 \mathrm{~nm}$ (a) before and (b) after Co/Pd multilayer film deposition 48

recording head that sets the direction of the magnetization of 100 grains to form a bit. To keep pace with the demand for increased data storage density, the magnetic bit size must be decreased with a commensurate decrease in grain size since, to maintain an adequate signal-to-noise ratio, the number of grains per bit is held approximately constant. However, as the individual grain volumes shrink, thermal activation can lead to unintended reversal of magnetization, since the thermal energy becomes competitive with the magnetic anisotropy energy of the grain. This is the superparamagnetic effect and it defines the grain volume for a given magnetic material, below which the recorded data can be lost due to thermal fluctuations. The magnetic storage industry is therefore seeking ways to delay the onset of superparamagnetism, and one possible route is to record the information in bit patterned media (BPM). A BPM consists of arrays of nanoscale magnetic islands, where each bit of information is stored in an individual island, thus the entire bit volume defines the magnetic anisotropy energy rather than the volume of individual grains. A number of methods have been employed to fabricate suitable nanomagnetic arrays, including electron beam lithography, facused ion beam lithography, and selfassembly methods 47 However, EUV-IL has particular advantages for the fabrication of BPM due to the possibility of creating high resolution structures over large areas with high spatial precision and high throughput.

EUV-IL is a relatively new technique for creating patterned magnetic thin films and, due to the short wavelengths of EUV, opens up the possibility of creating arrays of nanostructures with periods corresponding to areal densities greater than $1 \mathrm{Tbit} / \mathrm{in}^{2}$, which is beyond the range of optical interference lithography. Initially, EUV-IL combined with electrodeposition was used to create arrays of nickel islands 48 and more recently we have developed a method to create $50 \mathrm{~nm}$ period arrays of islands of $\mathrm{Co} / \mathrm{Pd}$ magnetic multilayer films, which have a perpendicular magnetic anisotropy suitable for bit patterned media 49 This latter fabrication process is schematically shown in Fig. 8 In a first step, a $30 \mathrm{~nm}$-thick HSQ resist is spin-coated on a silicon substrate. Following EUV-IL exposure through a grating mask comprised of four linear gratings, the HSQ resist is then developed in a tetramethyl ammonium hydroxide solution to produce an array of $30 \mathrm{~nm}$-high $\mathrm{SiO}_{x}$ pillars [Fig. 8(a)]. The magnetic film is then deposited on the prepatterned substrate by dc magnetron sputtering to create arrays of magnetic islands on top of the $\mathrm{SiO}_{x}$ pillars [Fig. 8(b)]. The pillars have a negative sidewall profile due to the strong absorption of EUV light by the HSQ film, so that the top receives a higher exposure dose than the bottom. This negative profile results in shadowing during film deposition, so that there is no magnetic material on the pillar sidewalls. This means that the magnetic islands are magnetically decoupled from the magnetic material in the trenches between the pillars, which is important if they are to be used for magnetic data storage. The growth of the magnetic film on the $\mathrm{SiO}_{x}$ pillars results in a mushroomlike structure (see schematic in Fig. 8). This lateral growth means that typically the size of the magnetic islands is larger than the original $\mathrm{SiO}_{x}$ pillars by a few nanometers, as seen in the SEM images of Fig. 8 The size of the magnetic islands can be tuned by changing the magnetic film thickness and to some extent, for a given mask, by changing the exposure dose values that control the original size of the $\mathrm{SiO}$ pillars. The $50 \mathrm{~nm}$ period magnetic islands shown in Fig. 8 are created over an area of $20 \times 20 \mu \mathrm{m}^{2}$ with a very narrow size distribution where the Gaussian sigma is less than $5 \%$, as analyzed using both atomic force microscopy (AFM) images and the SEM images.

Future work will focus on fabricating even smaller magnetic islands. Very recently it has been shown that dot arrays in calixarene resist with periods of $25 \mathrm{~nm}$ are achievable with EUV-IL 21 This dot period is equivalent to a data storage density of $1 \mathrm{Tbit} / \mathrm{in}^{2}$, a key milestone in the data storage roadmap.

\section{Chemical Patterning of Self-Assembled Monolayers: Applications in Nanobiotechnology}

Self-assembled monolayers (SAMs) are highly ordered organic films with a thickness of one molecule formed on surfaces by chemical adsorption and intermolecular forces 50 By using molecules with appropriate functional groups, SAMs form on almost all solid surfaces, providing a versatile route to their functionalization. Usually the formation of a SAM leads to a uniformly covered surface. However, molecular micro-/nanopatterns with specific chemical composition and shape are highly desirable for various research fields and applications, which include phase transitions in wetting layer 51 and polymer blends 17 hydrodynamic instabilities, 52 micro-/nanofluidics devices, 53 immobilization of nanoparticles 54 molecules 55 and fabrication of biological chips 56 Therefore, many lithographic techniques have been developed for SAMs patterning, 50 such as microcontact printing 57 photon 58 or electron irradiation, 55 and scanning-probe-based techniques 59 Lithographic techniques are often employed to induce damage of the exposed regions, which are then exchanged with another SAM of the desired functionality 175960 In some cases, however, a controlled modification of SAM 555961 is possible, which can directly be used to create chemical patterns. We have shown that EUV-IL 6263 presents an example 
(a)

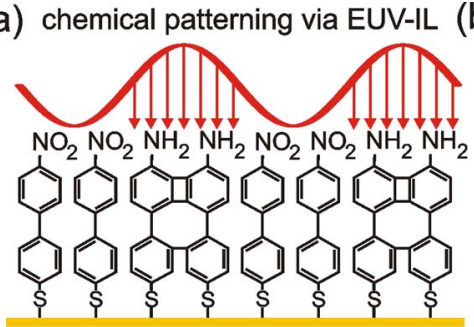

(c)

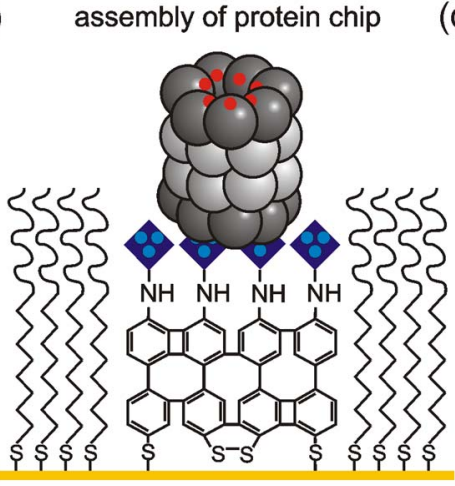

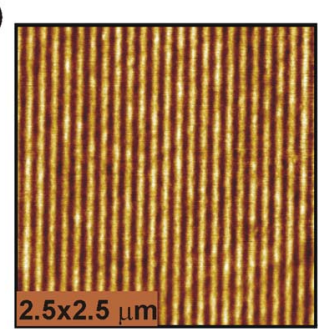

(d)

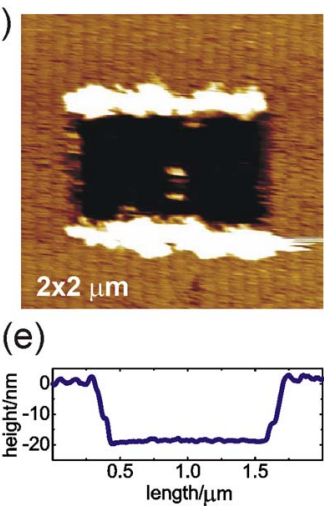

Fig. 9 (a) Representation of EUV-IL induced chemical patterning of nitro-1, 1'-biphenyl-4-thiol self-assembled monolayer on gold. EUV irradiation induces both the conversion of the terminal nitro groups into amino groups and the lateral cross-linking of the monolayer. (b) AFM micrograph (tapping mode, phase image) of the EUV-IL induced $100 \mathrm{~nm}$ periodic nitro/amino line pattern (light/dark lines). (c) Representation of the protein chip assembly on the prepatterned nitro/amino template. Multivalent chelators (dark blue squares) are grown on the amino-terminated areas and loaded with $\mathrm{Ni}$ (II) (light blue circles) to immobilize His-tagged (red circles) 20 S proteasome complex $(11 \times 15 \mathrm{~nm})$. Pristine areas of the NBPT SAM are exchanged with a protein repellent polyethyleneglycol SAM. (d) Topographic AFM image of oriented and specifically immobilized proteasome complexes (period $100 \mathrm{~nm}$ ); a rectangular area of proteins has been removed from the chip surface in AFM lithography mode (tapping mode, $z$-scale corresponds to $35 \mathrm{~nm}$ ). (e) Line profile across the area with and without proteins. For more detail, see Refs. 63 and 65 (Reprinted with permission from Refs. 63 and 65 copyright Wiley-VCH Verlag GmbH and Company). (Color online only.)

of such techniques. We have also demonstrated the utilization of the generated chemical patterns in nanobiotechnology.

EUV irradiation of a $4^{\prime}$-nitro-1,1'-biphenyl-4-thiol (NBPT) SAM on gold induces both the conversion of the terminal nitro groups of NBPT into amino groups and the lateral cross-linking of the underlying aromatic cores, resulting in the formation of an extended supramolecular network ${ }^{63}$ (Fig. 9p. This transformation was studied by $\mathrm{x}$-ray photoelectron spectroscopy, and the secondary electrons, generated in the substrate by EUV light, were identified to be the most likely cause of the observed changes. The complete transformation of nitro into amino groups and the lateral cross-linking occurs at typical radiation doses of $\sim 20 \mathrm{~J} / \mathrm{cm}^{2}$ for the NBPT SAM on gold substrates. Large-area $\left(\sim 4 \mathrm{~mm}^{2}\right)$ chemical line and dot patterns with periods from $2 \mu \mathrm{m}$ down to $60 \mathrm{~nm}$ consisting of the amino-terminated cross-linked areas and pristine NBPT areas have been generated using EUV-IL. An example of a $100 \mathrm{~nm}$ period nitro/amino line pattern is shown in Fig. 9(b) Considering the ultimate resolution of EUV-IL, low

proximity effects, and $\sim 1 \mathrm{~nm}$ thickness of the resist (NBPT SAM), we believe that the resolution of the chemical patterns can be further improved, and is at present limited by the roughness of the polycrystalline AuPd substrates used and the efficiency of the current exposure masks.

Highly parallel nitro/amino patterns on gold substrates present a versatile tool for the generation of functionally and chemically diverse molecular micro-/nanopatterns. To this end, the terminal amino-groups of the cross-linked areas can be further functionalized, whereas pristine areas of the NBPT SAM can he exchanged with a new SAM of desired functionality 63 This is of particular interest in medical diagnostics and nanobiotechnology for the generation of so-called protein chips $\underset{56}{ }$ i.e., solid substrates with specific molecular patterns used for functionally and laterally defined immobilization of proteins and protein complexes. Protein chips facilitate studies of protein functions and interactions which are of key importance for research in proteomics 64 Figure $9(\mathrm{c})$ schematically presents such a protein chip assembly 5 Here, multivalent chelators [cumulated $N$-nitrilotriacetic acid (NTA) moietie 66] were chemically grafted to EUV-IL prepatterned cross-linked amino terminated areas. Since the cross-linked areas show an exceptional stability in solution, the areas of the pristine NBPT SAM can be exchanged with a protein-repellent polyethyleneglycol SAM to prevent unspecific adsorption of proteins $[3$ Loading metal ions [e.g., $\mathrm{Ni}(\mathrm{II})]$ to the multivalent chelators results in laterally defined, specific, and reversible binding sides for His-tagged proteins. Figure 9(d) shows an AFM image of a $100 \mathrm{~nm}$ periodic line pattern of immobilized proteins 65 which was fabricated on the EUV-IL prepatterned NBPT SAM template presented in Fig. 9(b) As a biological model system, the His-tagged barrel-shaped 20S proteasome complex (with lateral dimensions of $11 \times 15 \mathrm{~nm}$ ) from the archaea Thermoplasma acidophilun 67 was employed. The His-tags were engineered to give an end-on orientation of proteins [Fig. 9(c)]. Specific and oriented immobilization of the His-tagged proteosome was demonstrated by mechanical removal of proteins from the chip surface with an AFM cantilever, and by complete regeneration of the chip surface ${ }^{65}$ The line profile over the region with proteasome and without proteasome reveals the height difference [Fig. 9(e)], which is in good qualitative agreement with its specific upright orientation.

The presented examples show the high potential of EUV-IL for chemical patterning of SAMs, which strongly facilitates the generation of diverse large area, highly parallel, and complex molecular micro-/nanopatterns. We expect that the lateral resolution of such patterns will further be improved so that they are capable of immobilization of single biological molecules. This will be a critical development for a manifold of research in molecular biology and nanobiotechnology, including single molecular studies in proteomics and cell adhesion phenomena.

\section{Radiation Grafting of Polymer Nanostructures}

In polymer technology, radiation grafting is a well known method to tune the properties of polymeric materials, in particular fluoropolymers 6 The polymer is exposed to particle or photon radiation with sufficient energy to break bonds in the backbone or side-chains of the polymer. The 


\section{EUV-IL exposure}

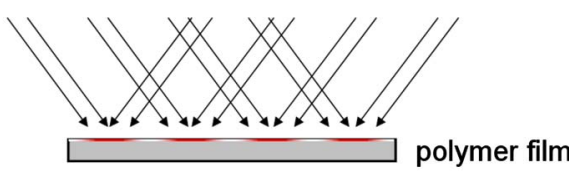

\section{2. polymer grafting}

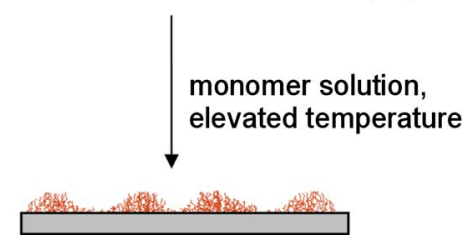

Fig. 10 Scheme for the polymer nanografting process: a fluoropolymer foil is first exposed to EUV radiation in the EUV-IL system, leading to a pattern of radicals at the surface of the foil. These radicals serve as initiators for the subsequent graft polymerization of a monomer, resulting in patterned polymer brushes at the surface.

radicals created in this step are then used as initiators for a graft polymerization reaction, in which polymer chains are formed step by step from the corresponding monomer. The graft reaction is carried out either during or after the exposure step. Depending on the parameters used, such as radiation energy, type and concentration of the monomer, type of solvent and reaction time, and temperature, radiation grafting can be used to modify bulk or surface properties of polymers.

EUV radiation is perfectly suited to create radicals for surface grafting of polymers. The photon energy is high enough to create a cascade of secondary electrons with sufficient energy to break chemical bonds, while the process is restricted to a few 10th nanometers near the surface due to the penetration length of EUV photons. The process used to create surface nanostructures with EUV-IL-based polymer grafting is schematically shown in Fig. 10. Typically, $100 \mu \mathrm{m}$-thick films of fluoropolymers such as [poly(ethylene-co-tetrafluoroethylene)] (ETFE), [poly (tetrafluoroethylene-co-hexafluoroproplyene)] (FEP), or [poly(vinylidene fluoride)] (PVDF) are exposed with EUVIL. The exposure dose determines the density of formed radicals, which in turn determines the density of polymer chains grown in the subsequent graft-polymerization step.

The EUV-radiation grafting differs in many aspects from other lithographic processes, which makes it attractive for a number of applications:
- It is an additive process, i.e., no sacrificial layers such as photoresists or intermediate metal masks are used.

- It can be carried out on polymer surfaces without the need for conducting layers.

- Structures made entirely from polymers are feasible in which the chemical properties of the grafted polymer can be very different from the base material.

Due to its intrinsic chemical amplification, i.e., long polymer chains are grown from single radicals, the process is very sensitive and needs a comparatively low exposure dose. For a number of monomers swch as acrylic acid, 69 styrene, 70 and various methacrylates, 71 a square root dependence between the exposure dose and the height of the grafted structures was found in the low dose range, indicating that the polymer chains are forced to stretch away from the surface and form so-called polymer brushes. In Fig. 11 the influence of the applied dose on the structure height is shown for two simultaneously grafted $284 \mathrm{~nm}$ period dot structures. This pronounced dose-height dependence can be employed to grow surface relief structures by spatial modulation of the exposure dose 70

The high rate of the polymerization reaction leads to growth of long polymer chains in short reaction times. Therefore, the overlapping of the polymer chains limits the spatial resolution to about $100 \mathrm{~nm}$. Improvements in the resolution down to the $50 \mathrm{~nm}$ range are possible by applying "living-radical" polymerization schemes, which limit the chain growth kinetics, or by postprocessing steps such as thermal or solvent annealing? 72

The firm anchoring of the grafted polymer chains enables various chemical derivatization reactions of the grafted chains, including biofunctionalization, e.g., by binding specific protein molecules. For example, the formation of biofunctional and thermoresponsive polymer brush structures has been demonstrated (Fig. 127071 Furthermore, the investigations of the growth behavior of the structures enahles new insight into surface polymerization mechanisms 7172

\section{Conclusions}

Since its introduction about a decade ago 10 EUV-IL has proven its unique potential as an enabling nanolithography tool. The diverse applications that are outlined in this work

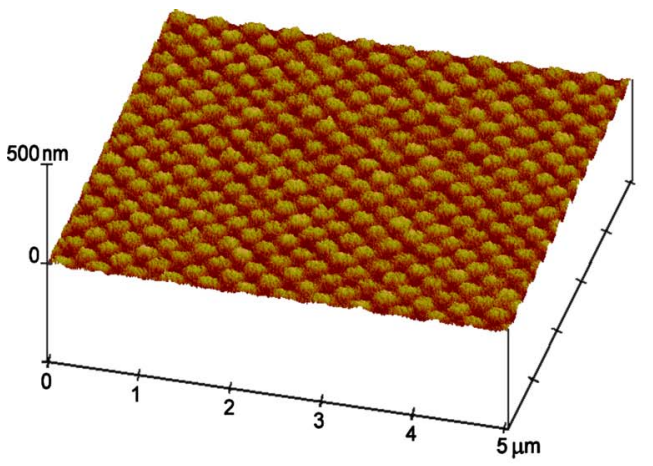

(a)

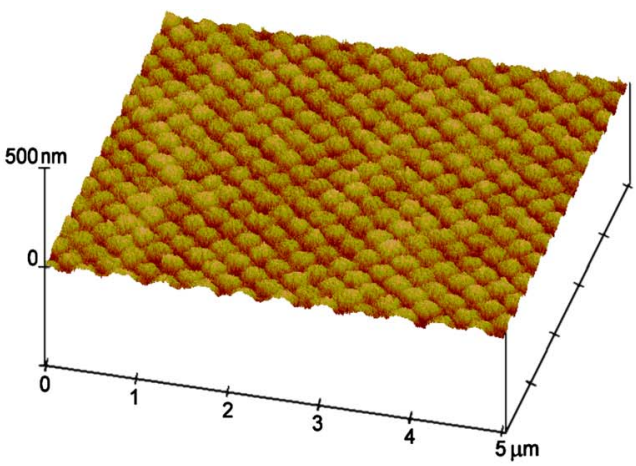

(b)

Fig. 11 AFM images of $142 \mathrm{~nm}$ half-pitch poly-(glycidyl methacrylate) brush structures grafted onto an ETFE substrate after EUV exposure. Increasing the dose from 5 to $20 \mathrm{~mJ} / \mathrm{cm}^{2}$ increases the height of the grafted dots from (a) $14 \mathrm{~nm}$ to (b) $21 \mathrm{~nm}$. 


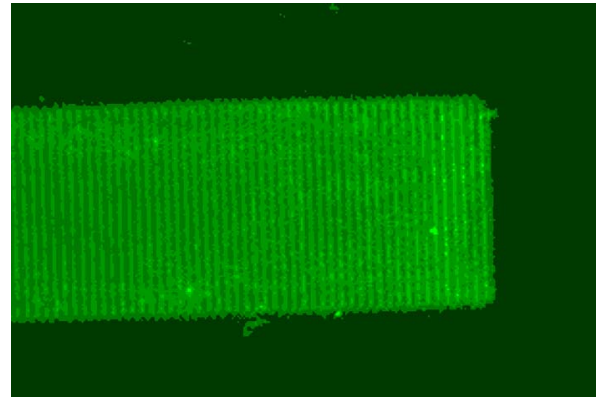

Fig. 12 Biofunctional microstructured polymer brush: fluorescence image of a $2-\mu \mathrm{m}$ period structure of poly-(glycidyl methacrylate) after reaction with biotin-pentylamine and binding of fluorescent labeled streptavidin.

demonstrate the versatility of the technique and the various ways it can help in diverse fields. For example, the unrivaled regularity and precision of the periodic structures created by EUV-IL is essential in the self-assembly field (e.g., for GeSi quantum dots) and fabrication of patterned magnetic media. The ability to expose nanopatterns on insulating samples is required for grafting on polymer substrates. Throughput and resolution are cross-cutting benefits of the technique, which help especially in fields like biology, where a great number of experiments need to be performed on similar substrates. With the recent demonstration of a resolution of $11 \mathrm{~nm}$ half-pitch patterns, we can expect EUV-IL to continue to serve scientific and technological applications for many years to come.

\section{Acknowledgments}

Heyderman, Thomson, and Luo are very grateful to Maggie Best and Olav Hellwig (Hitachi GST) for their fruitful collaboration on the magnetic dot arrays. Financial support by the European Community through the STREP project MAFIN (number 026513) is gratefully acknowledged. Auzelyte and Solak thank collaborators Janos Vörös, Ralph Spolenak, Brigitte Staedler, Robert McKenzie, and the Swiss CCMX program for funding the work on metallic nanowires. Part of this work was performed at the Swiss Light Source, Paul Scherrer Institut, Villigen, Switzerland.

\section{References}

1. G. C. Bjorklund_S_E_Harris, and J. F. Young, "Vacuum ultraviolet holography," Appl. Phys. Lett. 25(8), 451-452 (1974).

2. M. Wei, D. T. Attwood, T. K. Gustafson, and E. H. Anderson, "Patterning a 50-nm period grating using soft X-ray spatial frequency multiplication," J. Vac. Sci. Technol. B 12(6), 3648-3652 (1994).

3. J. Wallace, Y. C. Cheng, A. Isoyan, Q. Leonard, M. Fisher, M. Green, J. Bisognano, P. F. Nealey, and F. Cerrina "A novel EUV exposure station for nanotechnology studies," Nucl. Instrum. Methods Phys. Res. A 582(1), 254-257 (2007).

4. H. H. Solak, "Nanolithography with coherent extreme ultraviolet light,"J. Phys. D: Appl. Phys. 39(10), R171-R188 (2007).

5. A. Ritucc1, A. Reale, P. Zuppella, L. Reale, P. Tucceri, G. Tomassetti, P. Bettotti, and L. Pavesi, "Interference lithography by a_soft X-ray laser beam: Nanopatterning on photoresists,"J. Appl. Phys. 102(3) 034313-034314 (2007).

6. M. Goldstein, S. H. Lee, Y. A. Shroff, P. J. Silverman, D. Williams, H. Park, M. A. Piestrup, and R. H. Pantell, "FEL Applications in EUV lithography," Proc. 2005 FEL Conf., pp. 422-425 (2005).

7. W. Banqiu and K Aiay, "Extreme ultraviolet lithography: a review," J. Vac. Sci. Technol. B 25(6), 1743-1761 (2007).

8. K. E. Gonsalves, M. Thiyagarajan, J. H. Choi, P. Zimmerman, F. Cerrina, P. Nealey, V. Golovkina, J. Wallace and N Batina "High performance resist for EUV lithography," Microelectron. Eng. 77(1),
27-35 (2005)

9. K. Petrillo, Y. Wei, R. Brainard, G. Denbeaux, D. Goldfarb, C. S Koay, J. Mackey, W. Montgomery, W. Pierson, T. Wallow, and O Wood. "Are extreme ultraviolet resists ready for the $32 \mathrm{~nm}$ node?" $J$. Vac. Sci. Technol. B 25(6), 2490-2495 (2007).

10. H. H. Solak, D. He, W. Li, and F. Cerrina, "Nanolithography using extreme ultraviolet lithooraphy interferometry: $19 \mathrm{~nm}$ lines and spaces," J. Vac. Sci. Technol. B 17(6), 3052-3057 (1999).

11. P. W. Wachulak, M. G. Capeluto, M. C. Marconi, C. S. Menoni, and J. J. Rocca, "Patterning of nano-scale arrays by table-top extreme ultraviolet laser interferometric lithography," Opt. Express 15, 34653469 (2007).

12. P. Wachulak, M. Grisham, S. Heinbuch, D. Martz, W. Rockward, D. Hill, J. Rocca, C. Menoni, E. Anderson, and M. Marconi, "Interferometric lithography with an amplitude division interferometer and a desktop extreme ultraviolet laser,'J. Opt. Soc. Am. B 25, B104-B107 (2008).

13. H. Shiotani, S. Suzuki, D. Gun Lee, P. Naulleau, Y. Fukushima, R. Ohnishi, T. Watanabe, and H. Kinoshita, "Dual grating interferometric lithography for 22-nm node,"Jpn. J. Appl. Phys. 47, 4881-4885 (2008).

14. D. H. Bilderback, P. Elleaume, and E. Weckert "Review of third and next generation synchrotron light sources,"J. Phys. B 38(9), S773S797 (2005).

15. H. H. Solak, C. David, J. Gobrecht, L. Wang and E Cerrina "Fourwave EUV interference lithography," Microelectron. Eng. 61-62, 77-82 (2002)

16. H. H. Solak, "Space-invariant multiple-beam achromatic EUV interference lithography," Microelectron. Eng. 78-79, 410-416 (2005).

17. H. H. Solak, C. David, J Gobrecht, V. Golovkina, F. Cerrina, S. O. Kim, and P. F. Nealey "sub-50 nm period patterns with EUV interference lithography," Microelectron. Eng. 67-68(1), 56-62 (2003).

18. "The International Technology Roadmap for Semiconductors," see http://www.itrs.net (2007).

19. R. Gronheid, H. H. Solak, Y. Ekinci, A. Jouve, and F. V. Roey, "Characterization of extreme ultraviolet resists with interference lithography," Microelectron. Eng. 83(4-9), 1103-1106 (2006)

20. A. M. Goethals, R. Gronheid, F. V. Roey $H$ H Solak and Y Ekinci "Progress in EUV resist performance," J. Photopolym. Sci. Technol. 19(4), 501-506 (2006)

21. H. H. Solak and Y. Ekinci, "Bit-array patterns with density over $1 \mathrm{Tbit} /$ in. [sup 2] fabricated by extreme ultraviolet interference lithography," J. Vac. Sci. Technol. B 25(6), 2123-2126 (2007).

22. H. H. Solak, I. Y. Ekinc, P. S. Kàser, and S. Park. "Photon-beam lithography reaches $12.5 \mathrm{~nm}$ half-pitch resolution,"J. Vac. Sci. Technol. B 25(1), 91-95 (2007)

23. Y. Ekinci, H. H. Solak, and C. David, "Extraordinary optical transmission in the ultraviolet region through aluminum hole arrays,"Opt. Lett. 32(2), 172-174 (2007).

24. Y. Ekinci, H. H. Solak, C. David, and H. Sigg, "BilayerAl_wire-grids as broadband and high-performance polarizers," Opt. Express 14(6) 2323-2324 (2006)

25. S. Park, H. Schift, H. H. Solak, and J. Gobrecht, "Stamps for nanoimprint lithography by extreme ultraviolet interference lithography," $J$. Vac. Sci. Technol. B 22, 3246-3250 (2004).

26. H. H. Solak, C. David, and J. Gobrecht, "Fabrication of highresolution zone plates with wideband extreme-ultraviolet holography," Appl. Phys. Lett. 85(14), 2700-2702 (2004).

27. S. S. Sarkar, P. K. Sahoo, H. H. Solak, C. David, and J. F. Van der Veen, "Fabrication of Fresnel zone plates by holography in the extreme ultraviolet region," J. Vac. Sci. Technol. B 26(6), 2160-2163 (2008)

28. M. P. Stoykovich, E. W. Edwards, H. H. Solak, and P. F. Nealey, "Phase behavior of symmetric ternary block copolymerhomopolvmer blends in thin films and on chemically patterned surfaces," Phys. Rev. Lett. 97, 147802 (2006)

29. F. Juillerat, H. H. Solak, P. Bowen, and H. Hofmann, "Fabrication of large-area ordered arrays of nanoparticles on patterned substrates," Nano/Micro Eng. Mole. Syst. NEMS '07 2nd IEEE Intl. Conf. 16, 1311-1316S (2005).

30. D. Grieshaber, R. Mackenzie, J. Vörös, and E. Reimhult "Electrochemical biosensors-sensor principles and architectures,"Sensors 8 1400-1458 (2008).

31. C. Durkan and M. E. Welland, "Size effects in the electrical resistivity of polycrystalline nanowires," Phys. Rev. B 61(20), 14215 (2000).

32. J. E. Green, J. Wook Choi, A. Bouka1, Y. Bunimovich, E. JohnstonHalperin, E. Deionno, Y. Luo, B. A. Sheriff, K. Xu, Y. Shik Shin, H.-R. Tseng, J. F. Stoddart, and J. R. Heath, “A 160-kilobit molecular electronic memory patterned at 1011 bits per square centimetre," Nature (London) 445(7126), 414-417 (2007).

33. B. Städler, H. H. Solak, S. Frerker, K. Bonroy, F. Frederix, J. Vörös, and H. M. Grandin. "Nanopatterning of gold colloids for label-free biosensing," Nanotechnology 18(15), 155306 (2007)

34. V. Auzelyte, H. H. Solak, Y. Ekinci, R. Mackenzie, J. Vörös, S. 
Olliges, and R. Snolenak, "Large area arrays of metal nanowires," Microelectron. Eng. 85(5-6), 1131-1134 (2008).

35. S. Olliges, P. A. Gruber, V. Auzelyte, Y. Ekinci, H. H. Solak, and R. Spolenak, "Tensile strength of gold_nanointerconnects without the influence of strain gradients,"'Acta Mater. 55(15), 5201-5210 (2007).

36. S. Olliges, P. A. Gruber, S. Orso, V. Auzelyte, Y. Ekinci, H. H. Solak, and R. Spolenak, "In situ observation of cracks in gold nanointerconnects on flexible substrates," Scr. Mater. 58(3), 175-178 (2008).

37. O. G. Schmidt, A. Rastelli, G. S. Kar, R. Songmuang, S. Kiravittaya, M. Stoffel, U. Denker, S. Stufler, A. Zrenner, D. Grützmacher, B. Y. Nguyen, and P. Wennekers, "Novel nanostructure architectures," Syst. Nanostruct. 25(2-3), 280-287 (2004).

38. R. Vrijen, E. Yablonovitch, K. Wang, H. W. Jiang, A. Balandin, V. Roychowdhury, and T. Mor, "Electron-spin-resonance transistors for quantum computing in silicon-germanium heterostructures," Phys. Rev. A 62(1), 012306 (2000)

39. T_L Wang "Issues of nanoelectronics: a possible roadmap," $J$ Nanosci. Nanotechnol. 2(3-4), 235-266 (2002).

40. U. G. Schmidt, N. Y. Jin-Phillipp, C. Lange, U. Denker, and K. Eberl, "Long-range ordered_lines of self-assembled Ge islands on a flat Si (001) surface," Appl. Phys. Lett. 77(25), 4139-4141 (2000).

41. Z. Zhong, A. Hallovic, T. Fromherz, F. Schaeffler, and G. Bauer, "Two-dimensional periodic positioning of self-assembled Ge islands on prepatterned Si (001) substrates," Appl. Phys. Lett. 82(26), 47794781 (2003).

42. C. Dais, H. H. Solak, Y. Ekinci, E. Müller, H. Sigg, and D. Grützmacher, "Ge quantum dot molecules and crystals: preparation and properties," Surf. Sci. 601(13), 2787-2791 (2007).

43. C. Dais, H. H. Solak, E. Müller, and D. Grützmacher, "Impact of template variations on shape and arrangement of $\mathrm{Si} / \mathrm{Ge}$ quantum dot arrays,"Appl. Phys. Lett. 92(14), 143102 (2008).

44. C. Dais, G. Mussler, H. Sigg, T. Fromherz, V. Auzelyte, H. H. Solak, and D. Grätzmacher, "Photoluminescence studies of SiGe quantum dot arrays pepared by templated self assembly," EPL 84, 67017 (2008).

45. D. Grützmacher, T. Fromherz, C. Dais, J. Stangl, E. Müller, Y. Ekinci, H. H. Solak, H. Sigg, R. T. Lechner, E. Wintersberger, S. Birner, V. Holy, and G.Bauer, "Three-dimensional Si/Ge quantum dot crystals," Nano Lett. 7(10), 3150-3156 (2007).

46. J. Tersotf, C. Teicher, and M. G. Lagally "Self-organization in growth of quantum dot superlattices," Phys. Rev. Lett. 76(10), 1675 1678 (1996)

47. B. D. Terris and T. Thomson, "Nanofabricated_and_self-assembled magnetic structures as data storage media," J. Phys. D 38, R199_ R222 (2005)

48. L. J. Heyderman, H. H. Solak, C. David, D. Atkinson, R. P. Cowburn, and F. Nolting, "Arrays of nanoscale magnetic dots: Eabrication by X-ray interference lithography and characterization," Appl. Phys. Lett. 85, 4989-4991 (2004).

49. F. Luo, L. J. Heyderman, H. H. Solak, T. Thomson, and M. E. Best, "Nanoscale perpendicular magnetic island arrays fabricated by extreme ultraviolet interference lithography," Appl. Phys. Lett. 92, 102505 (2007)

50. J. C. Love, L. A. Estroff, J. K. Kriebel, R. G. Nuzzo, and G. M. Whitesides, "Self-assembled monolayers of thiolates on metals as a form of nanotechnology," Chem. Rev. (Washington, D.C.) 105(4), 1103-1169 (2005).

51. P. Lenz and R. Lipowsky, "Morphological transitions of wetting layers on structured surfaces,"Phys. Rev. Lett. 80(9), 1920-1923 (1998)

52. H. Gau, S. Herminghaus, P. Lenz, and R. Lipowsky, "Liquid morphologies on structured surfaces: from microchannels to microchips," Science 283(5398), 46-49 (1999).

53. Atencia and D. J. Beebe, "Controlled microfluidic interfaces," $\mathrm{Na}$ ture (London) 437(7059), 648-655 (2005)

54. P.M. Mendes, S. Jacke, K. Critchley, J. Plaza, Y. Chen, K. Nikitin, R. E. Palmer, J. A. Preece, S. D. Evans, and D. Fitzmaurice, "Gold nanoparticle patterning of silicon wafers using chemical e-beam lithography,"Langmuir 20(9), 3766-3768 (2004).

55. A. Gölzhäuser, W. Eck, W. Geyer, V. Stadler, T. Weimann, P. Hinze, and M. Grunze, "Chemical nanolithography with electron beams," Adv. Mater. Res. 13(11), 803-806 (2001).

56. K. L. Christman, V. D. Enriquez-Rios, and H. D. Maynard, "Nanopatterning proteins and peptides,"Soft Mater. 2(11), 928-939 (2006)

57. B. D. Gates, Q. B. Xu, J. C. Love, D. B Wolfe and G. M. Whitesides, "Unconventional nanofabrication," Annu. Rev. Mater. Res. 34, 339-372 (2004).

58. M. J. Tarlov, D. R. F. Burgess, and G. Gillen, "UV photopatterning of alkanethiolate monolayers self-assembled on gold and silver," J. Am. Chem. Soc. 115(12), 5305-5306 (1993).

59. S. Krämer, R. R. Fuierer, and C. B. Gorman "Scanning probe lithography using self-assembled monolayers," Chem. Rev. (Washington, D.C.) 103(11), 4367-4418 (2003).

60. S. Sun and G. J. Leggett, "Matching the resolution of electron beam lithography by scanning near-field photolithography," Nano Lett. 4(8), 1381-1384 (2004).

61. S. Q. Sun, M. Montague, K. Critchley, M. S. Chen, W. J. Dressick, S D. Evans, and G. J. Leggett, "Fabrication of biological nanostructures by scanning near-field photolithography of chloromethylphenyisiloxane monolayers," Nano Lett. 6(1), 29-33 (2006).

62. X. M. Yang, R. D. Peters, T. K. Kim, and P. F. Nealey, "Patterning of self-assembled monolayers with lateral dimensions of $0.15 \mathrm{mu}$ m using advanced lithography," J. Vac. Sci. Technol. B 17(6), 3203-3207 (1999)

63. A. Turchanin, M. Schnietz, M. El-Desawy, H. H. Solak, C. David, and A. Gölzhäuser, "Fabrication of molecular nanotemplates in selfassembled monolayers by extreme-ultraviolet-induced chemical lithography," Small 3(12), 2114-2119 (2007).

64. H.Zhu and M. Snyder, "Protein chip technology," Curr. Opin. Chem. Biol. 7(1), 55-63 (2003).

65. A. Turchanin, A. Tinazli, M. El-Desawy, H. Grossann, M. Schnietz, H. H. Solak, R. Tampé, and A. Gölzhäuser, "Molecular selfassembly, chemical lithography, and biochemical tweezers: a path fo the fabrication of functional nanometer-scale protein arrays," $A d v$. Mater. (Weinheim, Ger.) 20(3), 471-477 (2008).

66. A. Tinazli, J. L. Tang, R. Valiokas, S. Picuric, S. Lata, J. Piehler, B Liedberg, and R. Tampe, "High-affinity chelator thiols for switchable and oriented immobilization of histidine-tagoed proteins: a generic platform for protein chip technologies," Chem.-Eur. J. 11(18), 52495259 (2005)

67. E. Seemüller, A. Lupas, D. Stock, J. Löwe, R. Huber, and W Baumeister, "Proteasome from thermoplasma-acidophilum-a threonine protease," Science 268(5210), 579-582 (1995).

68. T. R. Dargaville, G. A. George, D. J. T. Hill, and A.K. Whittaker, "High energy radiation grafting of fluoropolymers," Prog. Polym. Sci. 28, 1355-1376 (2003)

69. H. P. Brack, C. Padeste, M. Slaski, S. Alkan, and H. H. Solak, "Preparation of micro- and nanopatterns of polymer chains grafted onto flexible polymer substrates," J. Am. Chem. Soc. 126(4), 1004 1005 (2004)

70. C. Padeste, P. Farquet, and H. H. Solak "Surface relief polymer structures grafted onto polymer films," Microelectron. Eng. 83(4-9), 1265-1268 (2006)

71. P. Farquet, C. Padeste, H. H. Solak, S. A. Gürsel, G. G. Scherer, and A. Wokaun, "EUV lithographic radiation orafting of thermoresponsive hydrogel nanostructures," Nucl. Instrum. Methods Phys. Res. B 265(1), 187-192 (2007).

72. P. Farquet, C. Padeste, H. H. Solak, S. A. Gürsel, G. G. Scherer, and A. Wokaun, "Extreme UV radiation grafting of glycidyl methacrylate nanostructures onto fluoropolymer foils by RAFT-mediated polymerization," Macromolecules 41, 6308-6316 (2008).

Biographes and photographs of the authors not available. 